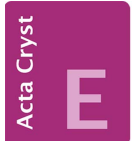
COMMUNICATIONS

ISSN 2056-9890

\section{Crystal structure of $(E)$-1-(4-tert-butyl- phenyl)-2-(4-iodophenyl)ethene}

\author{
Zhiwei Chen $^{\mathrm{a}}$ and Graeme J. Moxey ${ }^{\mathrm{a}, \mathrm{b}_{*}}$
}

${ }^{a}$ School of Chemical and Material Engineering, Jiangnan University, Wuxi, 214122, People's Republic of China, and ${ }^{\mathbf{b}}$ Research School of Chemistry, Australian National University, Canberra, ACT 2601, Australia. *Correspondence e-mail:

Graeme.Moxey@anu.edu.au

Received 1 April 2015; accepted 10 April 2015

Edited by H. Stoeckli-Evans, University of Neuchâtel, Switzerland

The title compound, $\mathrm{C}_{18} \mathrm{H}_{19} \mathrm{I}$, crystallized with two independent molecules $(A$ and $B)$ in the asymmetric unit. Both molecules have an $E$ conformation about the bridging $\mathrm{C}=\mathrm{C}$ bond. They differ in the orientation of the two benzene rings; the dihedral angle being $12.3(5)^{\circ}$ in molecule $A$, but only $1.0(6)^{\circ}$ in molecule $B$. In the crystal, the individual molecules are linked by $\mathrm{C}-\mathrm{I} \cdots \pi$ interactions forming zigzag $A$ and zigzag $B$ chains propagating along [001]. The structure was refined as an inversion twin [Flack parameter $=0.48(2)$ ].

Keywords: crystal structure; stilbene; iodoarene; C $-1 \cdots \pi$ interactions.

CCDC reference: 1053466

\section{Related literature}

For the syntheses of arylalkynes by Sonogashira crosscoupling of iodoarenes, see: Takahashi et al. (1980). For desilylation of the resultant trialkylsilylethynylarenes and the use of ethynylarenes in the construction of metal alkynyl complexes with enhanced non-linear optical properties, see: McDonagh et al. (1996a,b, 2003); Garcia et al. (2002). For related structures, see: Marras et al. (2006); Mariaca et al. (2009).

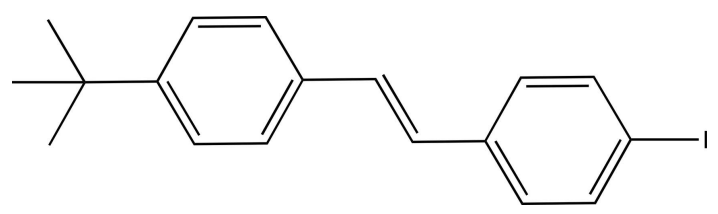

\section{Experimental}

2.1. Crystal data

$\mathrm{C}_{18} \mathrm{H}_{19} \mathrm{I} \quad M_{r}=362.23$
Orthorhombic, $P_{\mathcal{A}} 2_{1}$

$a=32.5385$ (9) $\AA$

$b=6.10513(15) \AA$

$c=15.8615(3) \AA$

$V=3150.91(14) \AA^{3}$

\subsection{Data collection}

Agilent SuperNova (Dual, $\mathrm{Cu}$ at zero, EosS2) diffractometer

Absorption correction: analytical (CrysAlis PRO; Agilent, 2014)

$T_{\min }=0.854, T_{\max }=0.966$

\subsection{Refinement}

$R\left[F^{2}>2 \sigma\left(F^{2}\right)\right]=0.050$

$w R\left(F^{2}\right)=0.133$

$S=1.04$

3770 reflections

350 parameters

67 restraints

$\mathrm{H}$-atom parameters constrained

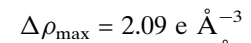

$\Delta \rho_{\min }=-1.31 \mathrm{e} \AA^{-3}$

Absolute structure: Flack (1983), 570 Friedel pairs

Absolute structure parameter: $0.48(2)$

Table 1

Hydrogen-bond geometry $\left(\AA{ }^{\circ}\right)$.

$C g 2$ and $C g 4$ are the centroids of the $\mathrm{C} 9-\mathrm{C} 14$ and $\mathrm{C} 27-\mathrm{C} 32$ rings, respectively.

\begin{tabular}{lllll}
\hline$D-\mathrm{H} \cdots A$ & $D-\mathrm{H}$ & $\mathrm{H} \cdots A$ & $D \cdots A$ & $D-\mathrm{H} \cdots A$ \\
\hline $\mathrm{C} 1-\mathrm{I} 1 \cdots C g 2^{\mathrm{i}}$ & $2.09(1)$ & $3.63(1)$ & $5.676(10)$ & $166(1)$ \\
$\mathrm{C} 19-\mathrm{I} 2 \cdots C g 4^{\mathrm{ii}}$ & $2.10(1)$ & $3.57(1)$ & $5.526(11)$ & $154(1)$ \\
\hline
\end{tabular}

Symmetry codes: (i) $-x+1,-y, z-\frac{1}{2}$; (ii) $-x+\frac{3}{2}, y-1, z+\frac{1}{2}$.

Data collection: CrysAlis PRO (Agilent, 2014); cell refinement: CrysAlis PRO; data reduction: CrysAlis PRO; program(s) used to solve structure: SHELXS97 (Sheldrick, 2008); program(s) used to refine structure: SHELXL2013 (Sheldrick, 2015); molecular graphics: OLEX2 (Dolomanov et al., 2009); software used to prepare material for publication: $O L E X 2$.

\section{Acknowledgements}

We gratefully acknowledge support from the Australian Research Council (LE130100057) to purchase the Agilent Technologies SuperNova and XCalibur diffractometers. We thank Professors C. Zhang (Jiangnan University), M. P. Cifuentes (Australian National University) and M. G. Humphrey (Australian National University) for assistance.

Supporting information for this paper is available from the IUCr electronic archives (Reference: SU5108).

\section{References}

Agilent (2014). CrysAlis PRO. Agilent Technologies, Yarnton, England. Dolomanov, O. V., Bourhis, L. J., Gildea, R. J., Howard, J. A. K. \& Puschmann, H. (2009). J. Appl. Cryst. 42, 339-341.

Flack, H. D. (1983). Acta Cryst. A39, 876-881.

Garcia, M. H., Robalo, M. P., Dias, A. R., Duarte, M. T., Wenseleers, W., Aerts, G., Goovaerts, E., Cifuentes, M. P., Hurst, S., Humphrey, M. G., Samoc, M. \& Luther-Davies, B. (2002). Organometallics, 21, 2107-2118.

Mariaca, R., Labat, G., Behrnd, N.-R., Bonin, M., Helbling, F., Eggli, P., Couderc, G., Neels, A., Stoeckli-Evans, H. \& Hulliger, J. (2009). J. Fluor. Chem. 130, 175-196. 


\section{data reports}

Marras, G., Metrangolo, P., Meyer, F., Pilati, T., Resnati, G. \& Vij, A. (2006). New J. Chem. 30, 1397-1402.

McDonagh, A. M., Powell, C. E., Morrall, J. P., Cifuentes, M. P. \& Humphrey, M. G. (2003). Organometallics, 22, 1402-1413.

McDonagh, A. M., Whittall, I. R., Humphrey, M. G., Hockless, D. C. R., Skelton, B. W. \& White, A. H. (1996a). J. Organomet. Chem., 523, 33-40.
McDonagh, A. M., Whittall, I. R., Humphrey, M. G., Skelton, B. W. \& White, A. H. (1996b). J. Organomet. Chem., 519, 229-235.

Sheldrick, G. M. (2008). Acta Cryst. A64, 112-122.

Sheldrick, G. M. (2015). Acta Cryst. C71, 3-8.

Takahashi, S., Kuroyama, Y., Sonogashira, K. \& Hagihara, N. (1980). Synthesis, 627-630. 


\section{supporting information}

Acta Cryst. (2015). E71, o309-o310 [https://doi.org/10.1107/S2056989015007185]

\section{Crystal structure of (E)-1-(4-tert-butylphenyl)-2-(4-iodophenyl)ethene}

\section{Zhiwei Chen and Graeme J. Moxey}

\section{S1. Synthesis and crystallization}

(E)-1-(4-tert-butylphenyl)-2-(4-bromophenyl)ethene (1.00 g, $3.17 \mathrm{mmol})$ was dissolved in distilled THF (40 $\mathrm{mL})$ and cooled to $195 \mathrm{~K}$ (liquid nitrogen bath) under $\mathrm{N}_{2}$ for $30 \mathrm{~min}$. BuLi (2.97 mL, $1.6 \mathrm{M}, 4.76 \mathrm{mmol}$ ) was added and the mixture was stirred for $2 \mathrm{~h}$. A solution of $\mathrm{I}_{2}(1.20 \mathrm{~g}, 4.76 \mathrm{mmol})$ in THF $(20 \mathrm{~mL})$ was then added and the reaction was allowed to warm to room temperature. A saturated solution of sodium thiosulfate $(10 \mathrm{~mL})$ and water $(20 \mathrm{~mL})$ were then added and the mixture stirred until clear. The mixture was then extracted with $\mathrm{CH}_{2} \mathrm{Cl}_{2}$, stirred over anhydrous $\mathrm{MgSO}_{4}$, filtered and taken to dryness to yield the title compound as a yellow solid. The solid was extracted with a small amount of $\mathrm{CH}_{2} \mathrm{Cl}_{2}$ and the extract was passed through a pad of silica with petrol as eluent. The eluate was reduced in volume, affording the title compound as a white solid (yield: $1.0 \mathrm{~g}, 87 \%$ ). The numbering scheme of the title compound for the NMR assignments is given in Fig. 3. ${ }^{1} \mathrm{H}-\mathrm{NMR}\left(400 \mathrm{MHz}, \mathrm{CDCl}_{3}\right): \delta 7.66\left(\mathrm{~d}, J_{\mathrm{HH}}=8 \mathrm{~Hz}, 2 \mathrm{H}, \mathrm{H}_{7}\right), 7.44\left(\mathrm{~d}, J_{\mathrm{HH}}=8 \mathrm{~Hz}\right.$, $\left.2 \mathrm{H}, \mathrm{H}_{3}\right), 7.38\left(\mathrm{~d}, J_{\mathrm{HH}}=8 \mathrm{~Hz}, 2 \mathrm{H}, \mathrm{H}_{2}\right), 7.23\left(\mathrm{~d}, J_{\mathrm{HH}}=8 \mathrm{~Hz}, 2 \mathrm{H}, \mathrm{H}_{6}\right), 7.09\left(\mathrm{~d}, J_{\mathrm{HH}}=16 \mathrm{~Hz}, 1 \mathrm{H}, \mathrm{H}_{4}\right), 6.97\left(\mathrm{~d}, J_{\mathrm{HH}}=16 \mathrm{~Hz}\right.$, $\left.1 \mathrm{H}, \mathrm{H}_{5}\right), 1.33\left(\mathrm{~s}, 9 \mathrm{H}, \mathrm{H}_{1}\right)$. Crystals suitable for X-ray diffraction analysis were obtained by slow evaporation of a solution in hexane.

\section{S2. Refinement}

Crystal data, data collection and structure refinement details are summarized below. The $\mathrm{H}$ atoms were included in calculated positions and treated as riding: $\mathrm{C}-\mathrm{H}=0.93-0.96 \AA$ with $\mathrm{U}_{\mathrm{iso}}(\mathrm{H})=1.5 \mathrm{U}_{\mathrm{eq}}(\mathrm{C})$ for methyl $\mathrm{H}$ atoms and $1.2 \mathrm{U}_{\mathrm{eq}}(\mathrm{C})$ for other $\mathrm{H}$ atoms. The structure was refined as an inversion twin: Flack parameter $=0.48$ (2). Rigid bond restraints (RIGU) were applied to atoms C15, C16, C17, C18, C22, C25, C26, C27, C28, C32, C33, C34, C35, C36. 


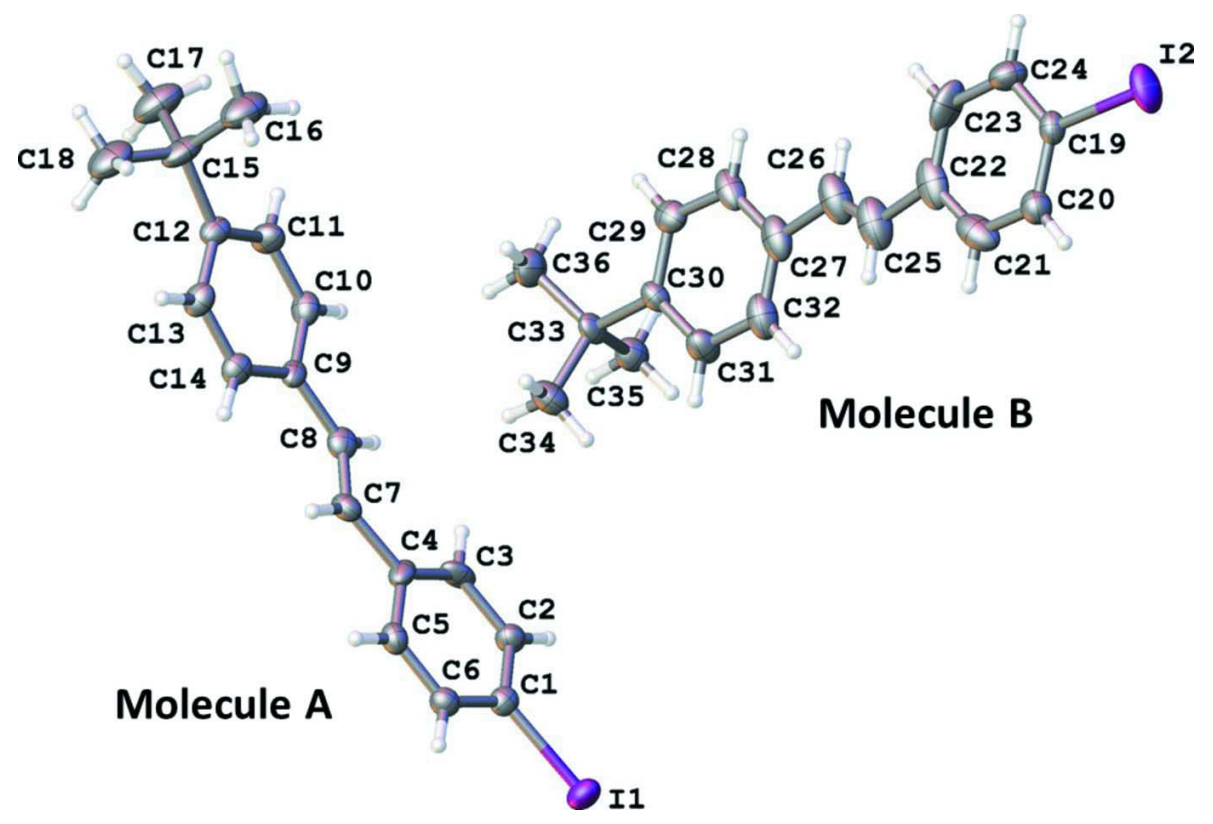

Figure 1

Molecular structure of the two independent molecules $(A$ and $B)$ of the title compound, with atom labelling. Displacement ellipsoids are drawn at the $40 \%$ probability level.

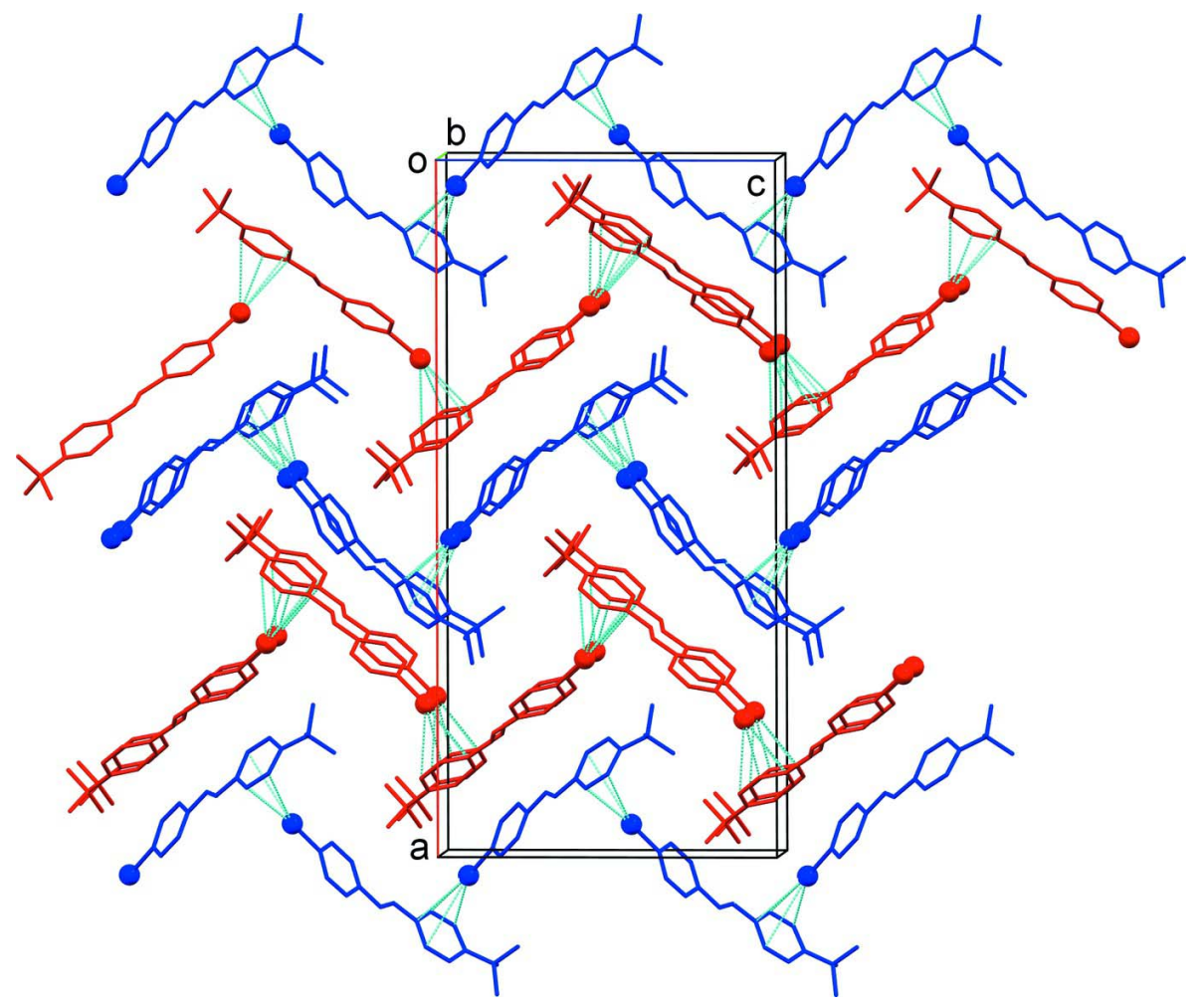

Figure 2

A view along the $b$ axis of the crystal packing of the title compound. The $\mathrm{C}-\mathrm{I} \cdots \pi$ interactions are represented as dashed lines (see Table 1 for details; molecule $A$ blue, molecule $B$ red). 


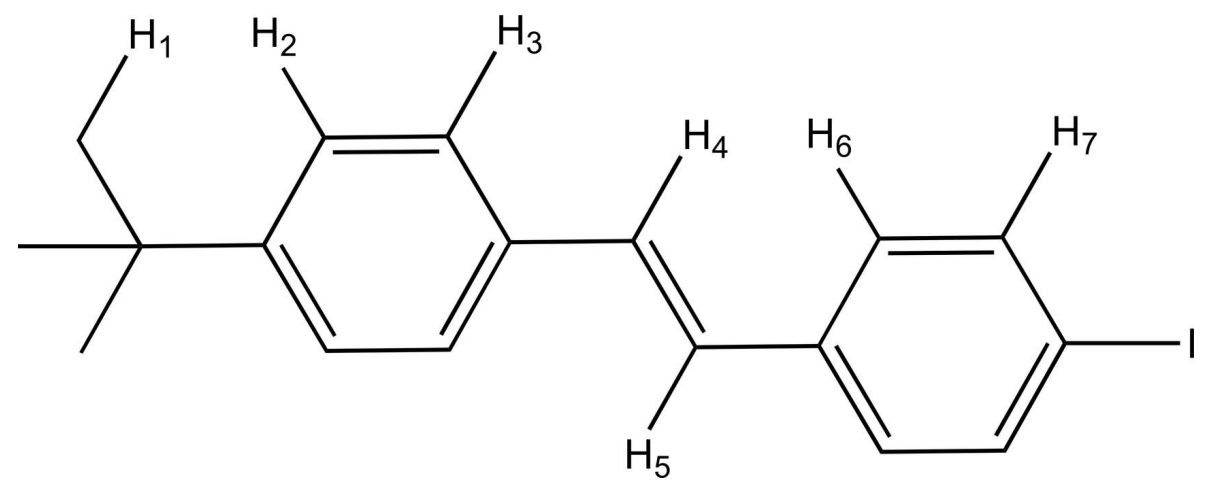

Figure 3

Atom numbering scheme of the title compound for ${ }^{1} \mathrm{H}$ NMR assignments.

(E)-1-(4-tert-Butylphenyl)-2-(4-iodophenyl)ethene

\section{Crystal data}

\section{$\mathrm{C}_{18} \mathrm{H}_{19} \mathrm{I}$}

$M_{r}=362.23$

Orthorhombic, $\mathrm{Pca} 2_{1}$

$a=32.5385(9) \AA$

$b=6.10513(15) \AA$

$c=15.8615(3) \AA$

$V=3150.91(14) \AA^{3}$

$Z=8$

$F(000)=1440$

\section{Data collection}

Agilent SuperNova (Dual, Cu at zero, EosS2) diffractometer

Radiation source: sealed X-ray tube, SuperNova (Cu) X-ray Source

Mirror monochromator

Detector resolution: 8.1297 pixels $\mathrm{mm}^{-1}$

$\omega$ scans

Absorption correction: analytical

(CrysAlis PRO; Agilent, 2014)

Refinement

Refinement on $F^{2}$

Least-squares matrix: full

$R\left[F^{2}>2 \sigma\left(F^{2}\right)\right]=0.050$

$w R\left(F^{2}\right)=0.133$

$S=1.04$

3770 reflections

350 parameters

67 restraints

Primary atom site location: structure-invariant direct methods

Secondary atom site location: difference Fourier map
$D_{\mathrm{x}}=1.527 \mathrm{Mg} \mathrm{m}^{-3}$

$\mathrm{Cu} K \alpha$ radiation, $\lambda=1.54184 \AA$

Cell parameters from 4062 reflections

$\theta=2.7-71.7^{\circ}$

$\mu=15.83 \mathrm{~mm}^{-1}$

$T=150 \mathrm{~K}$

Needle, colourless

$0.16 \times 0.05 \times 0.02 \mathrm{~mm}$

$T_{\min }=0.854, T_{\max }=0.966$

10293 measured reflections

3770 independent reflections

3559 reflections with $I>2 \sigma(I)$

$R_{\text {int }}=0.033$

$\theta_{\text {max }}=72.3^{\circ}, \theta_{\text {min }}=3.9^{\circ}$

$h=-36 \rightarrow 40$

$k=-7 \rightarrow 7$

$l=-7 \rightarrow 19$

Hydrogen site location: inferred from neighbouring sites

$\mathrm{H}$-atom parameters constrained

$w=1 /\left[\sigma^{2}\left(F_{\mathrm{o}}^{2}\right)+(0.0776 P)^{2}+7.7934 P\right]$

where $P=\left(F_{\mathrm{o}}^{2}+2 F_{\mathrm{c}}^{2}\right) / 3$

$(\Delta / \sigma)_{\max }=0.001$

$\Delta \rho_{\max }=2.09 \mathrm{e} \AA^{-3}$

$\Delta \rho_{\min }=-1.31$ e $\AA^{-3}$

Absolute structure: Flack (1983), 570 Friedel pairs

Absolute structure parameter: 0.48 (2) 


\section{Special details}

Geometry. All e.s.d.'s (except the e.s.d. in the dihedral angle between two 1.s. planes) are estimated using the full covariance matrix. The cell e.s.d.'s are taken into account individually in the estimation of e.s.d.'s in distances, angles and torsion angles; correlations between e.s.d.'s in cell parameters are only used when they are defined by crystal symmetry. An approximate (isotropic) treatment of cell e.s.d.'s is used for estimating e.s.d.'s involving 1.s. planes.

Refinement. Refined as a 2-component inversion twin.

Fractional atomic coordinates and isotropic or equivalent isotropic displacement parameters $\left(\AA^{2}\right)$

\begin{tabular}{|c|c|c|c|c|}
\hline & $x$ & $y$ & $z$ & $U_{\text {iso }} * / U_{\text {eq }}$ \\
\hline I1 & $0.54022(2)$ & $-0.31330(10)$ & $0.04996(5)$ & $0.04638(19)$ \\
\hline $\mathrm{C} 1$ & $0.4990(3)$ & $-0.1418(15)$ & $0.1266(6)$ & $0.0337(19)$ \\
\hline $\mathrm{C} 2$ & $0.5086(3)$ & $0.0691(15)$ & $0.1547(7)$ & $0.0366(19)$ \\
\hline $\mathrm{H} 2$ & 0.5327 & 0.1383 & 0.1375 & $0.044^{*}$ \\
\hline $\mathrm{C} 3$ & $0.4807(4)$ & $0.1750(14)$ & $0.2101(7)$ & $0.041(2)$ \\
\hline $\mathrm{H} 3$ & 0.4870 & 0.3146 & 0.2297 & $0.049^{*}$ \\
\hline $\mathrm{C} 4$ & $0.4457(3)$ & $0.0801(18)$ & $0.2351(6)$ & $0.038(2)$ \\
\hline $\mathrm{C} 5$ & $0.4356(3)$ & $-0.1290(17)$ & $0.2036(6)$ & $0.0361(19)$ \\
\hline H5 & 0.4110 & -0.1953 & 0.2195 & $0.043^{*}$ \\
\hline C6 & $0.4621(3)$ & $-0.2362(16)$ & $0.1489(7)$ & $0.036(2)$ \\
\hline H6 & 0.4549 & -0.3723 & 0.1272 & $0.043^{*}$ \\
\hline $\mathrm{C} 7$ & $0.4158(3)$ & $0.1806(16)$ & $0.2943(7)$ & $0.036(2)$ \\
\hline H7 & 0.3932 & 0.0972 & 0.3102 & $0.043^{*}$ \\
\hline $\mathrm{C} 8$ & $0.4191(3)$ & $0.3824(17)$ & $0.3267(6)$ & $0.038(2)$ \\
\hline $\mathrm{H} 8$ & 0.4402 & 0.4695 & 0.3060 & $0.046^{*}$ \\
\hline C9 & $0.3926(3)$ & $0.4803(15)$ & $0.3914(5)$ & $0.0314(18)$ \\
\hline C10 & $0.4042(3)$ & $0.6749(16)$ & $0.4264(7)$ & $0.040(2)$ \\
\hline H10 & 0.4268 & 0.7489 & 0.4045 & $0.048^{*}$ \\
\hline C11 & $0.3829(3)$ & $0.7647(17)$ & $0.4941(8)$ & $0.043(2)$ \\
\hline H11 & 0.3918 & 0.8970 & 0.5168 & $0.051 *$ \\
\hline $\mathrm{C} 12$ & $0.3485(3)$ & $0.6614(15)$ & $0.5290(6)$ & $0.035(2)$ \\
\hline $\mathrm{C} 13$ & $0.3352(3)$ & $0.4714(16)$ & $0.4881(7)$ & $0.040(2)$ \\
\hline H13 & 0.3114 & 0.4025 & 0.5067 & $0.048^{*}$ \\
\hline $\mathrm{C} 14$ & $0.3563(3)$ & $0.3835(15)$ & $0.4213(6)$ & $0.037(2)$ \\
\hline H14 & 0.3464 & 0.2575 & 0.3953 & $0.044 *$ \\
\hline $\mathrm{C} 15$ & $0.3286(5)$ & $0.743(2)$ & $0.6106(8)$ & $0.056(3)$ \\
\hline $\mathrm{C} 16$ & $0.3539(6)$ & $0.657(3)$ & $0.6841(10)$ & 0.077 (3) \\
\hline H16A & 0.3519 & 0.5001 & 0.6862 & $0.116^{*}$ \\
\hline H16B & 0.3437 & 0.7178 & 0.7359 & $0.116^{*}$ \\
\hline $\mathrm{H} 16 \mathrm{C}$ & 0.3821 & 0.6985 & 0.6768 & $0.116^{*}$ \\
\hline $\mathrm{C} 17$ & $0.3247(6)$ & 0.985 & $0.6122(10)$ & $0.077(4)$ \\
\hline H17A & 0.3148 & 1.0351 & 0.5586 & $0.115^{*}$ \\
\hline H17B & 0.3510 & 1.0488 & 0.6236 & $0.115^{*}$ \\
\hline $\mathrm{H} 17 \mathrm{C}$ & 0.3056 & 1.0266 & 0.6556 & $0.115^{*}$ \\
\hline $\mathrm{C} 18$ & $0.2857(5)$ & $0.652(3)$ & $0.6225(10)$ & $0.071(3)$ \\
\hline H18A & 0.2873 & 0.5082 & 0.6469 & $0.107^{*}$ \\
\hline H18B & 0.2721 & 0.6435 & 0.5689 & $0.107^{*}$ \\
\hline $\mathrm{H} 18 \mathrm{C}$ & 0.2704 & 0.7463 & 0.6594 & $0.107^{*}$ \\
\hline
\end{tabular}




$\begin{array}{lllll}\mathrm{I} 2 & 0.78793(2) & -0.28068(15) & 0.94298(6) & 0.0660(3) \\ \mathrm{C} 19 & 0.7487(3) & -0.1295(17) & 0.8555(7) & 0.037(2) \\ \mathrm{C} 20 & 0.7411(3) & -0.230(2) & 0.7792(8) & 0.046(3) \\ \mathrm{H} 20 & 0.7518 & -0.3680 & 0.7677 & 0.055^{*} \\ \mathrm{C} 21 & 0.7175(4) & -0.124(3) & 0.7204(8) & 0.064(4) \\ \mathrm{H} 21 & 0.7135 & -0.1904 & 0.6684 & 0.077^{*} \\ \mathrm{C} 22 & 0.6996(4) & 0.073(3) & 0.7341(10) & 0.063(4) \\ \mathrm{C} 23 & 0.7063(4) & 0.169(2) & 0.8102(12) & 0.066(4) \\ \mathrm{H} 23 & 0.6939 & 0.3027 & 0.8217 & 0.079^{*} \\ \mathrm{C} 24 & 0.7315(4) & 0.072(2) & 0.8725(8) & 0.053(3) \\ \mathrm{H} 24 & 0.7363 & 0.1425 & 0.9236 & 0.063^{*} \\ \mathrm{C} 25 & 0.6767(4) & 0.164(3) & 0.6608(12) & 0.072(3) \\ \mathrm{H} 25 & 0.6724 & 0.0661 & 0.6169 & 0.086^{*} \\ \mathrm{C} 26 & 0.6631(4) & 0.339(3) & 0.6495(11) & 0.067(3) \\ \mathrm{H} 26 & 0.6681 & 0.4387 & 0.6927 & 0.080^{*} \\ \mathrm{C} 27 & 0.6392(3) & 0.429(2) & 0.5768(8) & 0.056(2) \\ \mathrm{C} 28 & 0.6210(3) & 0.629(2) & 0.5896(7) & 0.049(2) \\ \mathrm{H} 28 & 0.6257 & 0.7007 & 0.6404 & 0.059^{*} \\ \mathrm{C} 29 & 0.5963(3) & 0.7274(18) & 0.5311(7) & 0.043(2) \\ \mathrm{H} 29 & 0.5840 & 0.8609 & 0.5437 & 0.051^{*} \\ \mathrm{C} 30 & 0.5893(3) & 0.6305(15) & 0.4530(7) & 0.0351(18) \\ \mathrm{C} 31 & 0.6083(3) & 0.4297(16) & 0.4386(8) & 0.048(2) \\ \mathrm{H} 31 & 0.6045 & 0.3588 & 0.3873 & 0.057^{*} \\ \mathrm{C} 32 & 0.6327(4) & 0.334(2) & 0.5003(10) & 0.057(3) \\ \mathrm{H} 32 & 0.6452 & 0.2004 & 0.4888 & 0.069^{*} \\ \mathrm{C} 33 & 0.5640(3) & 0.7429(16) & 0.3844(7) & 0.0379(18) \\ \mathrm{C} 34 & 0.5377(4) & 0.578(2) & 0.3364(8) & 0.055(3) \\ \mathrm{H} 34 \mathrm{~A} & 0.5198 & 0.5036 & 0.3752 & 0.082^{*} \\ \mathrm{H} 34 \mathrm{~B} & 0.5551 & 0.4729 & 0.3091 & 0.082^{*} \\ \mathrm{H} 34 \mathrm{C} & 0.5215 & 0.6532 & 0.2949 & 0.082^{*} \\ \mathrm{C} 35 & 0.5934(4) & 0.8569(19) & 0.3232(8) & 0.047(2) \\ \mathrm{H} 35 \mathrm{~A} & 0.6122 & 0.7514 & 0.3003 & 0.070^{*} \\ \mathrm{H} 35 \mathrm{~B} & 0.6085 & 0.9683 & 0.3526 & 0.070^{*} \\ \mathrm{H} 35 \mathrm{C} & 0.5780 & 0.9226 & 0.2783 & 0.070^{*} \\ \mathrm{C} 36 & 0.5344(4) & 0.911(2) & 0.4202(9) & 0.055(3) \\ \mathrm{H} 36 \mathrm{~A} & 0.5171 & 0.8429 & 0.4616 & 0.083^{*} \\ \mathrm{H} 36 \mathrm{~B} & 0.5177 & 0.9693 & 0.3756 & 0.083^{*} \\ \mathrm{H} 36 \mathrm{C} & 0.5497 & 1.0278 & 0.4460 & 0.083^{*} \\ & & & & \end{array}$

Atomic displacement parameters $\left(\AA^{2}\right)$

\begin{tabular}{lllllll}
\hline & $U^{11}$ & $U^{22}$ & $U^{33}$ & $U^{12}$ & $U^{13}$ & $U^{23}$ \\
\hline I1 & $0.0447(3)$ & $0.0457(3)$ & $0.0487(3)$ & $0.0059(2)$ & $0.0084(3)$ & $-0.0103(3)$ \\
C1 & $0.037(5)$ & $0.033(4)$ & $0.032(4)$ & $0.003(4)$ & $0.000(4)$ & $0.005(4)$ \\
C2 & $0.038(4)$ & $0.032(4)$ & $0.040(5)$ & $0.000(4)$ & $-0.002(4)$ & $-0.002(4)$ \\
C3 & $0.058(6)$ & $0.019(4)$ & $0.045(6)$ & $0.001(4)$ & $-0.020(5)$ & $-0.007(4)$ \\
C4 & $0.035(5)$ & $0.058(6)$ & $0.021(4)$ & $0.014(4)$ & $0.003(4)$ & $-0.001(4)$ \\
C5 & $0.029(4)$ & $0.042(5)$ & $0.037(5)$ & $-0.003(4)$ & $-0.003(4)$ & $0.004(4)$
\end{tabular}




\begin{tabular}{|c|c|c|c|c|c|c|}
\hline C6 & $0.038(5)$ & $0.033(4)$ & $0.036(5)$ & -0.001 (4) & $-0.003(4)$ & $0.000(4)$ \\
\hline $\mathrm{C} 7$ & $0.036(5)$ & $0.043(5)$ & $0.030(5)$ & $-0.007(4)$ & -0.004 & -0.002 \\
\hline $\mathrm{C} 8$ & $0.039(5)$ & $0.043(5)$ & $0.032(4)$ & $0.003(4)$ & -0.003 & 0.010 \\
\hline C9 & $0.029(4)$ & $0.044(5)$ & $0.020(4)$ & $0.007(4)$ & 0.001 (3) & 0.005 \\
\hline $\mathrm{C} 10$ & $0.032(4)$ & $0.048(5)$ & $0.039(5)$ & $0.000(4)$ & $0.005(4)$ & $0.010(4)$ \\
\hline C11 & $0.045(5)$ & $0.036(4)$ & $0.048(6)$ & $-0.007(4)$ & $0.004(5)$ & -0.007 (4) \\
\hline $\mathrm{C} 12$ & $0.042(5)$ & $0.038(4)$ & $0.026(5)$ & $0.008(4)$ & $0.004(4)$ & 0.005 \\
\hline $\mathrm{C} 13$ & $0.037(4)$ & $0.037(4)$ & $0.044(5)$ & $-0.002(4)$ & $0.011(4)$ & $0.005(4)$ \\
\hline $\mathrm{C} 14$ & $0.041(5)$ & $0.032(4)$ & $0.038(5)$ & $0.005(4)$ & $0.001(4)$ & $0.006(4)$ \\
\hline $\mathrm{C} 15$ & $0.072(6)$ & $0.056(5)$ & $0.040(5)$ & $0.019(4)$ & $0.023(4)$ & -0.003 \\
\hline $\mathrm{C} 16$ & $0.096(7)$ & $0.086(8)$ & $0.049(5)$ & $0.027(6)$ & $0.016(5)$ & $-0.004(5)$ \\
\hline $\mathrm{C} 17$ & $0.097(8)$ & $0.065(5)$ & $0.069(8)$ & $0.019(5)$ & $0.034(7)$ & -0.003 (4) \\
\hline $\mathrm{C} 18$ & $0.080(6)$ & $0.082(7)$ & $0.052(7)$ & $0.014(5)$ & $0.027(5)$ & $-0.001(6)$ \\
\hline I2 & 0.0478 (4) & $0.0800(5)$ & $0.0701(6)$ & -0.0002 & $-0.0213(4)$ & $0.0277(5)$ \\
\hline $\mathrm{C} 19$ & $0.029(4)$ & $0.041(5)$ & $0.040(5)$ & $0.001(4)$ & $-0.001(4)$ & $0.007(4)$ \\
\hline $\mathrm{C} 20$ & $0.040(6)$ & $0.052(6)$ & $0.046(6)$ & $0.000(5)$ & $-0.002(5)$ & $0.002(5)$ \\
\hline $\mathrm{C} 21$ & $0.067(8)$ & $0.089(10)$ & $0.036(6)$ & $-0.029(8)$ & $-0.001(5)$ & $0.006(7)$ \\
\hline $\mathrm{C} 22$ & $0.037(5)$ & $0.082(8)$ & $0.069(7)$ & $-0.017(5)$ & $-0.003(5)$ & $0.039(6)$ \\
\hline $\mathrm{C} 23$ & $0.057(7)$ & $0.040(6)$ & $0.101(13)$ & $0.023(5)$ & $0.018(8)$ & 0.025 \\
\hline $\mathrm{C} 24$ & $0.063(7)$ & $0.049(6)$ & $0.047(6)$ & $0.006(5)$ & $0.005(6)$ & $-0.011(5)$ \\
\hline $\mathrm{C} 25$ & $0.051(6)$ & $0.086(6)$ & $0.079(7)$ & $-0.014(4)$ & $-0.011(5)$ & $0.037(5)$ \\
\hline $\mathrm{C} 26$ & $0.047(5)$ & $0.082(5)$ & $0.072(6)$ & $-0.016(4)$ & $-0.013(5)$ & $0.034(4)$ \\
\hline $\mathrm{C} 27$ & $0.034(4)$ & $0.076(5)$ & $0.058(4)$ & $-0.014(4)$ & $-0.003(3)$ & $0.026(4)$ \\
\hline $\mathrm{C} 28$ & $0.035(4)$ & $0.076(5)$ & $0.036(4)$ & $-0.012(4)$ & $-0.003(4)$ & $0.024(4)$ \\
\hline $\mathrm{C} 29$ & $0.039(5)$ & $0.051(5)$ & $0.039(6)$ & -0.008 & $0.008(4)$ & $0.002(4)$ \\
\hline $\mathrm{C} 30$ & $0.034(4)$ & $0.037(4)$ & $0.034(5)$ & -0.006 & $-0.007(4)$ & $0.004(4)$ \\
\hline $\mathrm{C} 31$ & $0.051(5)$ & $0.036(4)$ & $0.056(6)$ & $0.000(4)$ & $-0.013(5)$ & $-0.002(5)$ \\
\hline $\mathrm{C} 32$ & $0.041(5)$ & $0.065(6)$ & $0.066(5)$ & -0.005 & -0.008 & $0.023(4)$ \\
\hline $\mathrm{C} 33$ & $0.040(4)$ & $0.036(3)$ & $0.037(4)$ & $0.006(3)$ & $-0.016(3)$ & $0.003(3)$ \\
\hline C34 & 0.059 & $0.057(5)$ & $0.048(5)$ & -0.007 (4) & -0.015 & $-0.002(4)$ \\
\hline $\mathrm{C} 35$ & $0.051(4)$ & $0.042(4)$ & $0.046(5)$ & $0.004(4)$ & $-0.009(4)$ & $0.003(4)$ \\
\hline C36 & $0.053(5)$ & $0.054(5)$ & $0.059(6)$ & $0.014(4)$ & -0.008 & $0.003(4)$ \\
\hline
\end{tabular}

Geometric parameters $\left(\stackrel{A}{\circ}{ }^{\circ}\right)$

\begin{tabular}{llll}
\hline $\mathrm{I} 1-\mathrm{C} 1$ & $2.091(10)$ & $\mathrm{I} 2-\mathrm{C} 19$ & $2.099(10)$ \\
$\mathrm{C} 1-\mathrm{C} 2$ & $1.398(13)$ & $\mathrm{C} 19-\mathrm{C} 20$ & $1.379(17)$ \\
$\mathrm{C} 1-\mathrm{C} 6$ & $1.379(14)$ & $\mathrm{C} 19-\mathrm{C} 24$ & $1.379(15)$ \\
$\mathrm{C} 2-\mathrm{H} 2$ & 0.9300 & $\mathrm{C} 20-\mathrm{H} 20$ & 0.9300 \\
$\mathrm{C} 2-\mathrm{C} 3$ & $1.420(16)$ & $\mathrm{C} 20-\mathrm{C} 21$ & $1.372(19)$ \\
$\mathrm{C} 3-\mathrm{H} 3$ & 0.9300 & $\mathrm{C} 21-\mathrm{H} 21$ & 0.9300 \\
$\mathrm{C} 3-\mathrm{C} 4$ & $1.339(16)$ & $\mathrm{C} 21-\mathrm{C} 22$ & $1.35(2)$ \\
$\mathrm{C} 4-\mathrm{C} 5$ & $1.410(15)$ & $\mathrm{C} 22-\mathrm{C} 23$ & $1.36(2)$ \\
$\mathrm{C} 4-\mathrm{C} 7$ & $1.485(14)$ & $\mathrm{C} 22-\mathrm{C} 25$ & $1.490(19)$ \\
$\mathrm{C} 5-\mathrm{H} 5$ & 0.9300 & $\mathrm{C} 23-\mathrm{H} 23$ & 0.9300 \\
$\mathrm{C} 5-\mathrm{C} 6$ & $1.386(15)$ & $\mathrm{C} 23-\mathrm{C} 24$ & $1.41(2)$ \\
$\mathrm{C} 6-\mathrm{H} 6$ & 0.9300 & $\mathrm{C} 24-\mathrm{H} 24$ & 0.9300 \\
$\mathrm{C} 7-\mathrm{H} 7$ & 0.9300 & $\mathrm{C} 25-\mathrm{H} 25$ & 0.9300
\end{tabular}




\begin{tabular}{|c|c|c|c|}
\hline $\mathrm{C} 7-\mathrm{C} 8$ & $1.339(15)$ & $\mathrm{C} 25-\mathrm{C} 26$ & $1.17(2)$ \\
\hline $\mathrm{C} 8-\mathrm{H} 8$ & 0.9300 & $\mathrm{C} 26-\mathrm{H} 26$ & 0.9300 \\
\hline $\mathrm{C} 8-\mathrm{C} 9$ & $1.467(13)$ & $\mathrm{C} 26-\mathrm{C} 27$ & $1.494(18)$ \\
\hline $\mathrm{C} 9-\mathrm{C} 10$ & $1.364(14)$ & $\mathrm{C} 27-\mathrm{C} 28$ & $1.37(2)$ \\
\hline $\mathrm{C} 9-\mathrm{C} 14$ & $1.402(13)$ & $\mathrm{C} 27-\mathrm{C} 32$ & $1.36(2)$ \\
\hline $\mathrm{C} 10-\mathrm{H} 10$ & 0.9300 & $\mathrm{C} 28-\mathrm{H} 28$ & 0.9300 \\
\hline $\mathrm{C} 10-\mathrm{C} 11$ & $1.391(15)$ & $\mathrm{C} 28-\mathrm{C} 29$ & $1.367(16)$ \\
\hline $\mathrm{C} 11-\mathrm{H} 11$ & 0.9300 & $\mathrm{C} 29-\mathrm{H} 29$ & 0.9300 \\
\hline $\mathrm{C} 11-\mathrm{C} 12$ & $1.399(15)$ & $\mathrm{C} 29-\mathrm{C} 30$ & $1.391(15)$ \\
\hline $\mathrm{C} 12-\mathrm{C} 13$ & $1.397(14)$ & $\mathrm{C} 30-\mathrm{C} 31$ & $1.391(14)$ \\
\hline $\mathrm{C} 12-\mathrm{C} 15$ & $1.532(14)$ & $\mathrm{C} 30-\mathrm{C} 33$ & $1.527(13)$ \\
\hline $\mathrm{C} 13-\mathrm{H} 13$ & 0.9300 & C31-H31 & 0.9300 \\
\hline $\mathrm{C} 13-\mathrm{C} 14$ & $1.372(15)$ & $\mathrm{C} 31-\mathrm{C} 32$ & $1.390(17)$ \\
\hline C14-H14 & 0.9300 & $\mathrm{C} 32-\mathrm{H} 32$ & 0.9300 \\
\hline $\mathrm{C} 15-\mathrm{C} 16$ & $1.52(2)$ & $\mathrm{C} 33-\mathrm{C} 34$ & $1.526(15)$ \\
\hline $\mathrm{C} 15-\mathrm{C} 17$ & $1.48(2)$ & $\mathrm{C} 33-\mathrm{C} 35$ & $1.530(16)$ \\
\hline $\mathrm{C} 15-\mathrm{C} 18$ & $1.51(2)$ & $\mathrm{C} 33-\mathrm{C} 36$ & $1.518(16)$ \\
\hline $\mathrm{C} 16-\mathrm{H} 16 \mathrm{~A}$ & 0.9600 & $\mathrm{C} 34-\mathrm{H} 34 \mathrm{~A}$ & 0.9600 \\
\hline $\mathrm{C} 16-\mathrm{H} 16 \mathrm{~B}$ & 0.9600 & $\mathrm{C} 34-\mathrm{H} 34 \mathrm{~B}$ & 0.9600 \\
\hline $\mathrm{C} 16-\mathrm{H} 16 \mathrm{C}$ & 0.9600 & $\mathrm{C} 34-\mathrm{H} 34 \mathrm{C}$ & 0.9600 \\
\hline C17-H17A & 0.9600 & C35-H35A & 0.9600 \\
\hline C17-H17B & 0.9600 & $\mathrm{C} 35-\mathrm{H} 35 \mathrm{~B}$ & 0.9600 \\
\hline C17-H17C & 0.9600 & $\mathrm{C} 35-\mathrm{H} 35 \mathrm{C}$ & 0.9600 \\
\hline $\mathrm{C} 18-\mathrm{H} 18 \mathrm{~A}$ & 0.9600 & $\mathrm{C} 36-\mathrm{H} 36 \mathrm{~A}$ & 0.9600 \\
\hline C18-H18B & 0.9600 & $\mathrm{C} 36-\mathrm{H} 36 \mathrm{~B}$ & 0.9600 \\
\hline $\mathrm{C} 18-\mathrm{H} 18 \mathrm{C}$ & 0.9600 & $\mathrm{C} 36-\mathrm{H} 36 \mathrm{C}$ & 0.9600 \\
\hline $\mathrm{C} 2-\mathrm{C} 1-\mathrm{I} 1$ & $120.2(7)$ & $\mathrm{C} 20-\mathrm{C} 19-\mathrm{I} 2$ & $119.6(8)$ \\
\hline $\mathrm{C} 6-\mathrm{C} 1-\mathrm{I} 1$ & $119.9(7)$ & $\mathrm{C} 20-\mathrm{C} 19-\mathrm{C} 24$ & $119.7(10)$ \\
\hline $\mathrm{C} 6-\mathrm{C} 1-\mathrm{C} 2$ & $119.9(9)$ & $\mathrm{C} 24-\mathrm{C} 19-\mathrm{I} 2$ & $120.7(9)$ \\
\hline $\mathrm{C} 1-\mathrm{C} 2-\mathrm{H} 2$ & 120.9 & $\mathrm{C} 19-\mathrm{C} 20-\mathrm{H} 20$ & 120.4 \\
\hline $\mathrm{C} 1-\mathrm{C} 2-\mathrm{C} 3$ & $118.2(9)$ & $\mathrm{C} 21-\mathrm{C} 20-\mathrm{C} 19$ & $119.1(12)$ \\
\hline $\mathrm{C} 3-\mathrm{C} 2-\mathrm{H} 2$ & 120.9 & $\mathrm{C} 21-\mathrm{C} 20-\mathrm{H} 20$ & 120.4 \\
\hline $\mathrm{C} 2-\mathrm{C} 3-\mathrm{H} 3$ & 119.0 & $\mathrm{C} 20-\mathrm{C} 21-\mathrm{H} 21$ & 118.2 \\
\hline $\mathrm{C} 4-\mathrm{C} 3-\mathrm{C} 2$ & $122.0(9)$ & $\mathrm{C} 22-\mathrm{C} 21-\mathrm{C} 20$ & $123.6(14)$ \\
\hline $\mathrm{C} 4-\mathrm{C} 3-\mathrm{H} 3$ & 119.0 & $\mathrm{C} 22-\mathrm{C} 21-\mathrm{H} 21$ & 118.2 \\
\hline $\mathrm{C} 3-\mathrm{C} 4-\mathrm{C} 5$ & $119.1(9)$ & $\mathrm{C} 21-\mathrm{C} 22-\mathrm{C} 23$ & $117.1(12)$ \\
\hline $\mathrm{C} 3-\mathrm{C} 4-\mathrm{C} 7$ & $124.4(10)$ & $\mathrm{C} 21-\mathrm{C} 22-\mathrm{C} 25$ & $115.0(16)$ \\
\hline $\mathrm{C} 5-\mathrm{C} 4-\mathrm{C} 7$ & $116.5(9)$ & $\mathrm{C} 23-\mathrm{C} 22-\mathrm{C} 25$ & $127.8(15)$ \\
\hline $\mathrm{C} 4-\mathrm{C} 5-\mathrm{H} 5$ & 119.9 & $\mathrm{C} 22-\mathrm{C} 23-\mathrm{H} 23$ & 118.9 \\
\hline $\mathrm{C} 6-\mathrm{C} 5-\mathrm{C} 4$ & $120.2(9)$ & $\mathrm{C} 22-\mathrm{C} 23-\mathrm{C} 24$ & $122.3(12)$ \\
\hline $\mathrm{C} 6-\mathrm{C} 5-\mathrm{H} 5$ & 119.9 & $\mathrm{C} 24-\mathrm{C} 23-\mathrm{H} 23$ & 118.9 \\
\hline $\mathrm{C} 1-\mathrm{C} 6-\mathrm{C} 5$ & $120.3(9)$ & $\mathrm{C} 19-\mathrm{C} 24-\mathrm{C} 23$ & $118.2(12)$ \\
\hline $\mathrm{C} 1-\mathrm{C} 6-\mathrm{H} 6$ & 119.8 & $\mathrm{C} 19-\mathrm{C} 24-\mathrm{H} 24$ & 120.9 \\
\hline $\mathrm{C} 5-\mathrm{C} 6-\mathrm{H} 6$ & 119.8 & $\mathrm{C} 23-\mathrm{C} 24-\mathrm{H} 24$ & 120.9 \\
\hline $\mathrm{C} 4-\mathrm{C} 7-\mathrm{H} 7$ & 117.6 & $\mathrm{C} 22-\mathrm{C} 25-\mathrm{H} 25$ & 114.7 \\
\hline $\mathrm{C} 8-\mathrm{C} 7-\mathrm{C} 4$ & $124.9(10)$ & $\mathrm{C} 26-\mathrm{C} 25-\mathrm{C} 22$ & $131(2)$ \\
\hline $\mathrm{C} 8-\mathrm{C} 7-\mathrm{H} 7$ & 117.6 & $\mathrm{C} 26-\mathrm{C} 25-\mathrm{H} 25$ & 114.7 \\
\hline
\end{tabular}




\begin{tabular}{|c|c|c|c|}
\hline $\mathrm{C} 7-\mathrm{C} 8-\mathrm{H} 8$ & 116.7 & $\mathrm{C} 25-\mathrm{C} 26-\mathrm{H} 26$ & 114.8 \\
\hline $\mathrm{C} 7-\mathrm{C} 8-\mathrm{C} 9$ & $126.6(10)$ & $\mathrm{C} 25-\mathrm{C} 26-\mathrm{C} 27$ & $130.5(18)$ \\
\hline $\mathrm{C} 9-\mathrm{C} 8-\mathrm{H} 8$ & 116.7 & $\mathrm{C} 27-\mathrm{C} 26-\mathrm{H} 26$ & 114.8 \\
\hline $\mathrm{C} 10-\mathrm{C} 9-\mathrm{C} 8$ & $118.4(9)$ & $\mathrm{C} 28-\mathrm{C} 27-\mathrm{C} 26$ & $115.9(14)$ \\
\hline $\mathrm{C} 10-\mathrm{C} 9-\mathrm{C} 14$ & $117.6(9)$ & $\mathrm{C} 32-\mathrm{C} 27-\mathrm{C} 26$ & $127.8(14)$ \\
\hline $\mathrm{C} 14-\mathrm{C} 9-\mathrm{C} 8$ & $124.0(9)$ & $\mathrm{C} 32-\mathrm{C} 27-\mathrm{C} 28$ & $116.3(11)$ \\
\hline $\mathrm{C} 9-\mathrm{C} 10-\mathrm{H} 10$ & 119.4 & $\mathrm{C} 27-\mathrm{C} 28-\mathrm{H} 28$ & 118.5 \\
\hline $\mathrm{C} 9-\mathrm{C} 10-\mathrm{C} 11$ & $121.2(9)$ & $\mathrm{C} 29-\mathrm{C} 28-\mathrm{C} 27$ & $123.0(12)$ \\
\hline $\mathrm{C} 11-\mathrm{C} 10-\mathrm{H} 10$ & 119.4 & $\mathrm{C} 29-\mathrm{C} 28-\mathrm{H} 28$ & 118.5 \\
\hline $\mathrm{C} 10-\mathrm{C} 11-\mathrm{H} 11$ & 119.1 & $\mathrm{C} 28-\mathrm{C} 29-\mathrm{H} 29$ & 119.6 \\
\hline $\mathrm{C} 10-\mathrm{C} 11-\mathrm{C} 12$ & $121.8(9)$ & $\mathrm{C} 28-\mathrm{C} 29-\mathrm{C} 30$ & $120.9(11)$ \\
\hline $\mathrm{C} 12-\mathrm{C} 11-\mathrm{H} 11$ & 119.1 & $\mathrm{C} 30-\mathrm{C} 29-\mathrm{H} 29$ & 119.6 \\
\hline $\mathrm{C} 11-\mathrm{C} 12-\mathrm{C} 15$ & $121.8(10)$ & $\mathrm{C} 29-\mathrm{C} 30-\mathrm{C} 33$ & $122.1(9)$ \\
\hline $\mathrm{C} 13-\mathrm{C} 12-\mathrm{C} 11$ & $116.0(9)$ & $\mathrm{C} 31-\mathrm{C} 30-\mathrm{C} 29$ & $116.6(10)$ \\
\hline $\mathrm{C} 13-\mathrm{C} 12-\mathrm{C} 15$ & $122.1(10)$ & $\mathrm{C} 31-\mathrm{C} 30-\mathrm{C} 33$ & $121.2(10)$ \\
\hline $\mathrm{C} 12-\mathrm{C} 13-\mathrm{H} 13$ & 119.0 & $\mathrm{C} 30-\mathrm{C} 31-\mathrm{H} 31$ & 119.7 \\
\hline $\mathrm{C} 14-\mathrm{C} 13-\mathrm{C} 12$ & $121.9(9)$ & $\mathrm{C} 32-\mathrm{C} 31-\mathrm{C} 30$ & $120.5(12)$ \\
\hline $\mathrm{C} 14-\mathrm{C} 13-\mathrm{H} 13$ & 119.0 & $\mathrm{C} 32-\mathrm{C} 31-\mathrm{H} 31$ & 119.7 \\
\hline C9- $14-\mathrm{H} 14$ & 119.4 & $\mathrm{C} 27-\mathrm{C} 32-\mathrm{C} 31$ & $122.6(13)$ \\
\hline $\mathrm{C} 13-\mathrm{C} 14-\mathrm{C} 9$ & $121.1(9)$ & $\mathrm{C} 27-\mathrm{C} 32-\mathrm{H} 32$ & 118.7 \\
\hline $\mathrm{C} 13-\mathrm{C} 14-\mathrm{H} 14$ & 119.4 & $\mathrm{C} 31-\mathrm{C} 32-\mathrm{H} 32$ & 118.7 \\
\hline $\mathrm{C} 16-\mathrm{C} 15-\mathrm{C} 12$ & $107.8(10)$ & $\mathrm{C} 30-\mathrm{C} 33-\mathrm{C} 35$ & $108.6(8)$ \\
\hline $\mathrm{C} 17-\mathrm{C} 15-\mathrm{C} 12$ & $112.0(11)$ & $\mathrm{C} 34-\mathrm{C} 33-\mathrm{C} 30$ & $111.2(9)$ \\
\hline $\mathrm{C} 17-\mathrm{C} 15-\mathrm{C} 16$ & $112.2(15)$ & $\mathrm{C} 34-\mathrm{C} 33-\mathrm{C} 35$ & $109.6(10)$ \\
\hline $\mathrm{C} 17-\mathrm{C} 15-\mathrm{C} 18$ & $106.5(13)$ & $\mathrm{C} 36-\mathrm{C} 33-\mathrm{C} 30$ & $112.3(9)$ \\
\hline $\mathrm{C} 18-\mathrm{C} 15-\mathrm{C} 12$ & $112.1(12)$ & $\mathrm{C} 36-\mathrm{C} 33-\mathrm{C} 34$ & $106.1(10)$ \\
\hline $\mathrm{C} 18-\mathrm{C} 15-\mathrm{C} 16$ & $106.1(13)$ & $\mathrm{C} 36-\mathrm{C} 33-\mathrm{C} 35$ & $109.0(9)$ \\
\hline $\mathrm{C} 15-\mathrm{C} 16-\mathrm{H} 16 \mathrm{~A}$ & 109.5 & $\mathrm{C} 33-\mathrm{C} 34-\mathrm{H} 34 \mathrm{~A}$ & 109.5 \\
\hline $\mathrm{C} 15-\mathrm{C} 16-\mathrm{H} 16 \mathrm{~B}$ & 109.5 & $\mathrm{C} 33-\mathrm{C} 34-\mathrm{H} 34 \mathrm{~B}$ & 109.5 \\
\hline $\mathrm{C} 15-\mathrm{C} 16-\mathrm{H} 16 \mathrm{C}$ & 109.5 & $\mathrm{C} 33-\mathrm{C} 34-\mathrm{H} 34 \mathrm{C}$ & 109.5 \\
\hline $\mathrm{H} 16 \mathrm{~A}-\mathrm{C} 16-\mathrm{H} 16 \mathrm{~B}$ & 109.5 & $\mathrm{H} 34 \mathrm{~A}-\mathrm{C} 34-\mathrm{H} 34 \mathrm{~B}$ & 109.5 \\
\hline $\mathrm{H} 16 \mathrm{~A}-\mathrm{C} 16-\mathrm{H} 16 \mathrm{C}$ & 109.5 & $\mathrm{H} 34 \mathrm{~A}-\mathrm{C} 34-\mathrm{H} 34 \mathrm{C}$ & 109.5 \\
\hline $\mathrm{H} 16 \mathrm{~B}-\mathrm{C} 16-\mathrm{H} 16 \mathrm{C}$ & 109.5 & $\mathrm{H} 34 \mathrm{~B}-\mathrm{C} 34-\mathrm{H} 34 \mathrm{C}$ & 109.5 \\
\hline $\mathrm{C} 15-\mathrm{C} 17-\mathrm{H} 17 \mathrm{~A}$ & 109.5 & $\mathrm{C} 33-\mathrm{C} 35-\mathrm{H} 35 \mathrm{~A}$ & 109.5 \\
\hline $\mathrm{C} 15-\mathrm{C} 17-\mathrm{H} 17 \mathrm{~B}$ & 109.5 & $\mathrm{C} 33-\mathrm{C} 35-\mathrm{H} 35 \mathrm{~B}$ & 109.5 \\
\hline $\mathrm{C} 15-\mathrm{C} 17-\mathrm{H} 17 \mathrm{C}$ & 109.5 & $\mathrm{C} 33-\mathrm{C} 35-\mathrm{H} 35 \mathrm{C}$ & 109.5 \\
\hline $\mathrm{H} 17 \mathrm{~A}-\mathrm{C} 17-\mathrm{H} 17 \mathrm{~B}$ & 109.5 & $\mathrm{H} 35 \mathrm{~A}-\mathrm{C} 35-\mathrm{H} 35 \mathrm{~B}$ & 109.5 \\
\hline $\mathrm{H} 17 \mathrm{~A}-\mathrm{C} 17-\mathrm{H} 17 \mathrm{C}$ & 109.5 & $\mathrm{H} 35 \mathrm{~A}-\mathrm{C} 35-\mathrm{H} 35 \mathrm{C}$ & 109.5 \\
\hline $\mathrm{H} 17 \mathrm{~B}-\mathrm{C} 17-\mathrm{H} 17 \mathrm{C}$ & 109.5 & $\mathrm{H} 35 \mathrm{~B}-\mathrm{C} 35-\mathrm{H} 35 \mathrm{C}$ & 109.5 \\
\hline $\mathrm{C} 15-\mathrm{C} 18-\mathrm{H} 18 \mathrm{~A}$ & 109.5 & $\mathrm{C} 33-\mathrm{C} 36-\mathrm{H} 36 \mathrm{~A}$ & 109.5 \\
\hline $\mathrm{C} 15-\mathrm{C} 18-\mathrm{H} 18 \mathrm{~B}$ & 109.5 & $\mathrm{C} 33-\mathrm{C} 36-\mathrm{H} 36 \mathrm{~B}$ & 109.5 \\
\hline $\mathrm{C} 15-\mathrm{C} 18-\mathrm{H} 18 \mathrm{C}$ & 109.5 & $\mathrm{C} 33-\mathrm{C} 36-\mathrm{H} 36 \mathrm{C}$ & 109.5 \\
\hline $\mathrm{H} 18 \mathrm{~A}-\mathrm{C} 18-\mathrm{H} 18 \mathrm{~B}$ & 109.5 & $\mathrm{H} 36 \mathrm{~A}-\mathrm{C} 36-\mathrm{H} 36 \mathrm{~B}$ & 109.5 \\
\hline $\mathrm{H} 18 \mathrm{~A}-\mathrm{C} 18-\mathrm{H} 18 \mathrm{C}$ & 109.5 & $\mathrm{H} 36 \mathrm{~A}-\mathrm{C} 36-\mathrm{H} 36 \mathrm{C}$ & 109.5 \\
\hline $\mathrm{H} 18 \mathrm{~B}-\mathrm{C} 18-\mathrm{H} 18 \mathrm{C}$ & 109.5 & $\mathrm{H} 36 \mathrm{~B}-\mathrm{C} 36-\mathrm{H} 36 \mathrm{C}$ & 109.5 \\
\hline $\mathrm{I} 1-\mathrm{C} 1-\mathrm{C} 2-\mathrm{C} 3$ & $-176.8(7)$ & $\mathrm{I} 2-\mathrm{C} 19-\mathrm{C} 20-\mathrm{C} 21$ & $-176.0(9)$ \\
\hline $\mathrm{I} 1-\mathrm{C} 1-\mathrm{C} 6-\mathrm{C} 5$ & $176.3(7)$ & $\mathrm{I} 2-\mathrm{C} 19-\mathrm{C} 24-\mathrm{C} 23$ & $178.2(9)$ \\
\hline
\end{tabular}




$\mathrm{C} 1-\mathrm{C} 2-\mathrm{C} 3-\mathrm{C} 4$
$\mathrm{C} 2-\mathrm{C} 1-\mathrm{C} 6-\mathrm{C} 5$
$\mathrm{C} 2-\mathrm{C} 3-\mathrm{C} 4-\mathrm{C} 5$
$\mathrm{C} 2-\mathrm{C} 3-\mathrm{C} 4-\mathrm{C} 7$
$\mathrm{C} 3-\mathrm{C} 4-\mathrm{C} 5-\mathrm{C} 6$
$\mathrm{C} 3-\mathrm{C} 4-\mathrm{C} 7-\mathrm{C} 8$
$\mathrm{C} 4-\mathrm{C} 5-\mathrm{C} 6-\mathrm{C} 1$
$\mathrm{C} 4-\mathrm{C} 7-\mathrm{C} 8-\mathrm{C} 9$
$\mathrm{C} 5-\mathrm{C} 4-\mathrm{C} 7-\mathrm{C} 8$
$\mathrm{C} 6-\mathrm{C} 1-\mathrm{C} 2-\mathrm{C} 3$
$\mathrm{C} 7-\mathrm{C} 4-\mathrm{C} 5-\mathrm{C} 6$
$\mathrm{C} 7-\mathrm{C} 8-\mathrm{C} 9-\mathrm{C} 10$
$\mathrm{C} 7-\mathrm{C} 8-\mathrm{C} 9-\mathrm{C} 14$
$\mathrm{C} 8-\mathrm{C} 9-\mathrm{C} 10-\mathrm{C} 11$
$\mathrm{C} 8-\mathrm{C} 9-\mathrm{C} 14-\mathrm{C} 13$
$\mathrm{C} 9-\mathrm{C} 10-\mathrm{C} 11-\mathrm{C} 12$
$\mathrm{C} 10-\mathrm{C} 9-\mathrm{C} 14-\mathrm{C} 13$
$\mathrm{C} 10-\mathrm{C} 11-\mathrm{C} 12-\mathrm{C} 13$
$\mathrm{C} 10-\mathrm{C} 11-\mathrm{C} 12-\mathrm{C} 15$
$\mathrm{C} 11-\mathrm{C} 12-\mathrm{C} 13-\mathrm{C} 14$
$\mathrm{C} 11-\mathrm{C} 12-\mathrm{C} 15-\mathrm{C} 16$
$\mathrm{C} 11-\mathrm{C} 12-\mathrm{C} 15-\mathrm{C} 17$
$\mathrm{C} 11-\mathrm{C} 12-\mathrm{C} 15-\mathrm{C} 18$
$\mathrm{C} 12-\mathrm{C} 13-\mathrm{C} 14-\mathrm{C} 9$
$\mathrm{C} 13-\mathrm{C} 12-\mathrm{C} 15-\mathrm{C} 16$
$\mathrm{C} 13-\mathrm{C} 12-\mathrm{C} 15-\mathrm{C} 17$
$\mathrm{C} 13-\mathrm{C} 12-\mathrm{C} 15-\mathrm{C} 18$
$\mathrm{C} 14-\mathrm{C} 9-\mathrm{C} 10-\mathrm{C} 11$
$\mathrm{C} 15-\mathrm{C} 12-\mathrm{C} 13-\mathrm{C} 14$

$-0.8(15)$
$-4.3(15)$
$-1.8(15)$
$178.6(10)$
$1.4(15)$
$3.7(16)$
$1.7(15)$
$-173.6(9)$
$-175.9(9)$
$3.9(14)$
$-179.0(9)$
$169.9(10)$
$-8.6(15)$
$-173.1(10)$
$173.0(9)$
$-0.6(17)$
$-5.5(14)$
$-4.3(16)$
$171.5(11)$
$4.2(15)$
$-80.3(15)$
$43.5(18)$
$163.3(11)$
$0.6(15)$
$95.2(15)$
$-140.9(13)$
$-21.2(16)$
$5.5(14)$
$-171.6(10)$

$\mathrm{C} 19-\mathrm{C} 20-\mathrm{C} 21-\mathrm{C} 22$
$\mathrm{C} 20-\mathrm{C} 19-\mathrm{C} 24-\mathrm{C} 23$
$\mathrm{C} 20-\mathrm{C} 21-\mathrm{C} 22-\mathrm{C} 23$
$\mathrm{C} 20-\mathrm{C} 21-\mathrm{C} 22-\mathrm{C} 25$
$\mathrm{C} 21-\mathrm{C} 22-\mathrm{C} 23-\mathrm{C} 24$
$\mathrm{C} 21-\mathrm{C} 22-\mathrm{C} 25-\mathrm{C} 26$
$\mathrm{C} 22-\mathrm{C} 23-\mathrm{C} 24-\mathrm{C} 19$
$\mathrm{C} 22-\mathrm{C} 25-\mathrm{C} 26-\mathrm{C} 27$
$\mathrm{C} 23-\mathrm{C} 22-\mathrm{C} 25-\mathrm{C} 26$
$\mathrm{C} 24-\mathrm{C} 19-\mathrm{C} 20-\mathrm{C} 21$
$\mathrm{C} 25-\mathrm{C} 22-\mathrm{C} 23-\mathrm{C} 24$
$\mathrm{C} 25-\mathrm{C} 26-\mathrm{C} 27-\mathrm{C} 28$
$\mathrm{C} 25-\mathrm{C} 26-\mathrm{C} 27-\mathrm{C} 32$
$\mathrm{C} 26-\mathrm{C} 27-\mathrm{C} 28-\mathrm{C} 29$
$\mathrm{C} 26-\mathrm{C} 27-\mathrm{C} 32-\mathrm{C} 31$
$\mathrm{C} 27-\mathrm{C} 28-\mathrm{C} 29-\mathrm{C} 30$
$\mathrm{C} 28-\mathrm{C} 27-\mathrm{C} 32-\mathrm{C} 31$
$\mathrm{C} 28-\mathrm{C} 29-\mathrm{C} 30-\mathrm{C} 31$
$\mathrm{C} 28-\mathrm{C} 29-\mathrm{C} 30-\mathrm{C} 33$
$\mathrm{C} 29-\mathrm{C} 30-\mathrm{C} 31-\mathrm{C} 32$
$\mathrm{C} 29-\mathrm{C} 30-\mathrm{C} 33-\mathrm{C} 34$
$\mathrm{C} 29-\mathrm{C} 30-\mathrm{C} 33-\mathrm{C} 35$
$\mathrm{C} 29-\mathrm{C} 30-\mathrm{C} 33-\mathrm{C} 36$
$\mathrm{C} 30-\mathrm{C} 31-\mathrm{C} 32-\mathrm{C} 27$
$\mathrm{C} 31-\mathrm{C} 30-\mathrm{C} 33-\mathrm{C} 34$
$\mathrm{C} 31-\mathrm{C} 30-\mathrm{C} 33-\mathrm{C} 35$
$\mathrm{C} 31-\mathrm{C} 30-\mathrm{C} 33-\mathrm{C} 36$
$\mathrm{C} 32-\mathrm{C} 27-\mathrm{C} 28-\mathrm{C} 29$
$\mathrm{C} 33-\mathrm{C} 30-\mathrm{C} 31-\mathrm{C} 32$

$-3(2)$

$0.1(18)$

1 (2)

$177.6(11)$

$1(2)$

$-168.3(16)$

$-2(2)$

$-178.2(12)$

8 (3)

$2.1(17)$

$-174.7(12)$

$168.0(16)$

$-11(2)$

$-176.4(10)$

177.1 (12)

$-2.1(16)$

$-1.8(17)$

0.7 (14)

$-176.3(9)$

$0.0(15)$

$-142.2(10)$

$97.2(11)$

$-23.5(14)$

$0.5(18)$

$41.0(14)$

$-79.7(12)$

$159.7(10)$

$2.6(16)$

$177.0(10)$

Hydrogen-bond geometry $\left(A,{ }^{\circ}\right)$

$\mathrm{Cg} 2$ and $\mathrm{Cg} 4$ are the centroids of the $\mathrm{C} 9-\mathrm{C} 14$ and $\mathrm{C} 27-\mathrm{C} 32$ rings, respectively.

\begin{tabular}{lllll}
\hline$D-\mathrm{H} \cdots A$ & $D-\mathrm{H}$ & $\mathrm{H} \cdots A$ & $D \cdots A$ & $D-\mathrm{H} \cdots A$ \\
\hline $\mathrm{C} 1-\mathrm{I} 1 \cdots C g 2^{\mathrm{i}}$ & $2.09(1)$ & $3.63(1)$ & $5.676(10)$ & $166(1)$ \\
$\mathrm{C} 19-\mathrm{I} 2 \cdots C g 4^{\mathrm{ii}}$ & $2.10(1)$ & $3.57(1)$ & $5.526(11)$ & $154(1)$ \\
\hline
\end{tabular}

Symmetry codes: (i) $-x+1,-y, z-1 / 2$; (ii) $-x+3 / 2, y-1, z+1 / 2$. 